\title{
Adjuvant or neoadjuvant therapy for operable esophagogastric cancer?
}

\author{
Sing Yu Moorcraft • Elizabeth C. Smyth • \\ David Cunningham
}

Received: 23 October 2013/Accepted: 8 February 2014/Published online: 18 March 2014

(c) The International Gastric Cancer Association and The Japanese Gastric Cancer Association 2014

\begin{abstract}
Esophagogastric cancer encompasses proximal squamous cell carcinoma of the esophagus, distal esophageal/junctional adenocarcinoma of the esophagus and gastric adenocarcinoma. These diseases have different etiologies, geographic incidences and biologies. This review mainly focuses on the treatment of operable esophagogastric adenocarcinoma. In Asia, adjuvant chemotherapy is commonly used for patients with gastric cancer following the landmark ACTS-GC trial. In contrast, perioperative chemotherapy is a standard of care in many Western countries based on the results of the MAGIC trial. Neoadjuvant chemotherapy is better tolerated than adjuvant therapy, and therefore dose intensity is likely to be maintained in a greater proportion of patients. In addition, neoadjuvant treatment can lead to tumor downstaging, increasing the likelihood of achieving a complete surgical resection. This may be particularly important in Western populations, as these patients often present with more advanced tumors than Asian patients. Adjuvant chemoradiotherapy is a standard treatment option in the USA for adenocarcinoma of the stomach or gastroesophageal junction as a result of the INT-0116 trial, but the benefit of this approach after a D2 resection has not been confirmed. Neoadjuvant chemoradiotherapy may reduce the risk of local recurrence and may be particularly beneficial for patients with squamous cell carcinoma as these tumors are more radiosensitive. However, patients with esophagogastric adenocarcinoma are more likely to relapse with distant disease, and therefore a systemic disease approach with
\end{abstract}

S. Y. Moorcraft $(\bowtie) \cdot$ E. C. Smyth · D. Cunningham Gastrointestinal Unit, Department of Medicine, The Royal Marsden NHS Foundation Trust, Sutton SM2 5PT, UK e-mail: singyu.moorcraft@rmh.nhs.uk chemotherapy is likely to be more beneficial than a purely localized treatment strategy for these patients.

Keywords Gastric cancer - Esophageal cancer · Chemotherapy $\cdot$ Chemoradiotherapy $\cdot$ Standard of care

\section{Introduction}

Esophagogastric cancer is a broad term, and despite similarities in treatment approaches this definition encompasses at least three (if not four) diseases each with a distinct etiology, geographic incidence and biology. Esophageal cancer incorporates both proximal radiosensitive squamous cell carcinoma (SCC) and distal esophageal/junctional adenocarcinoma. Adenocarcinoma of the gastroesophageal junction (GEJ), although treated and staged similarly to cancers of the proximal esophagus, is significantly less radiosensitive and is biologically more closely related to gastric adenocarcinoma. In a similar manner, true gastric adenocarcinoma may be subdivided by Lauren's histology and molecular profiling into intestinal and diffuse type cancers, each of which differs in terms of presentation, chemosensitivity and patterns of recurrence (although this does not currently impact on management) [1-3]. Thus, when adopting an evidence-based approach for the optimal perioperative management for a patient with any of these cancers, it is essential to consider the proportions of patients of each histological subtype and anatomical subsite enrolled in any study as this may significantly influence results. A second major factor that must be reflected on is the geographic location where the trial was conducted as this may affect stage at presentation, surgical outcomes and treatment tolerability. 


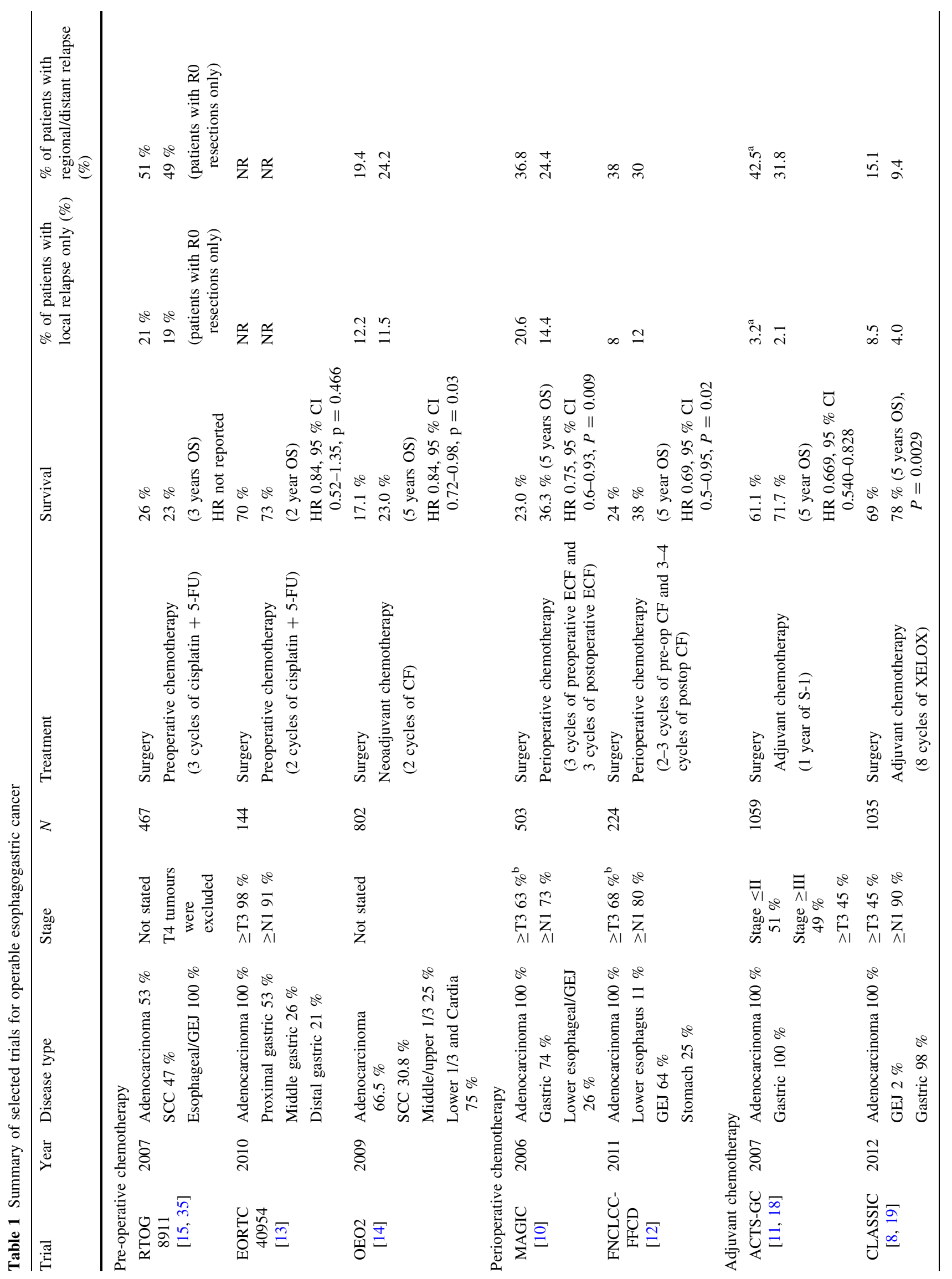




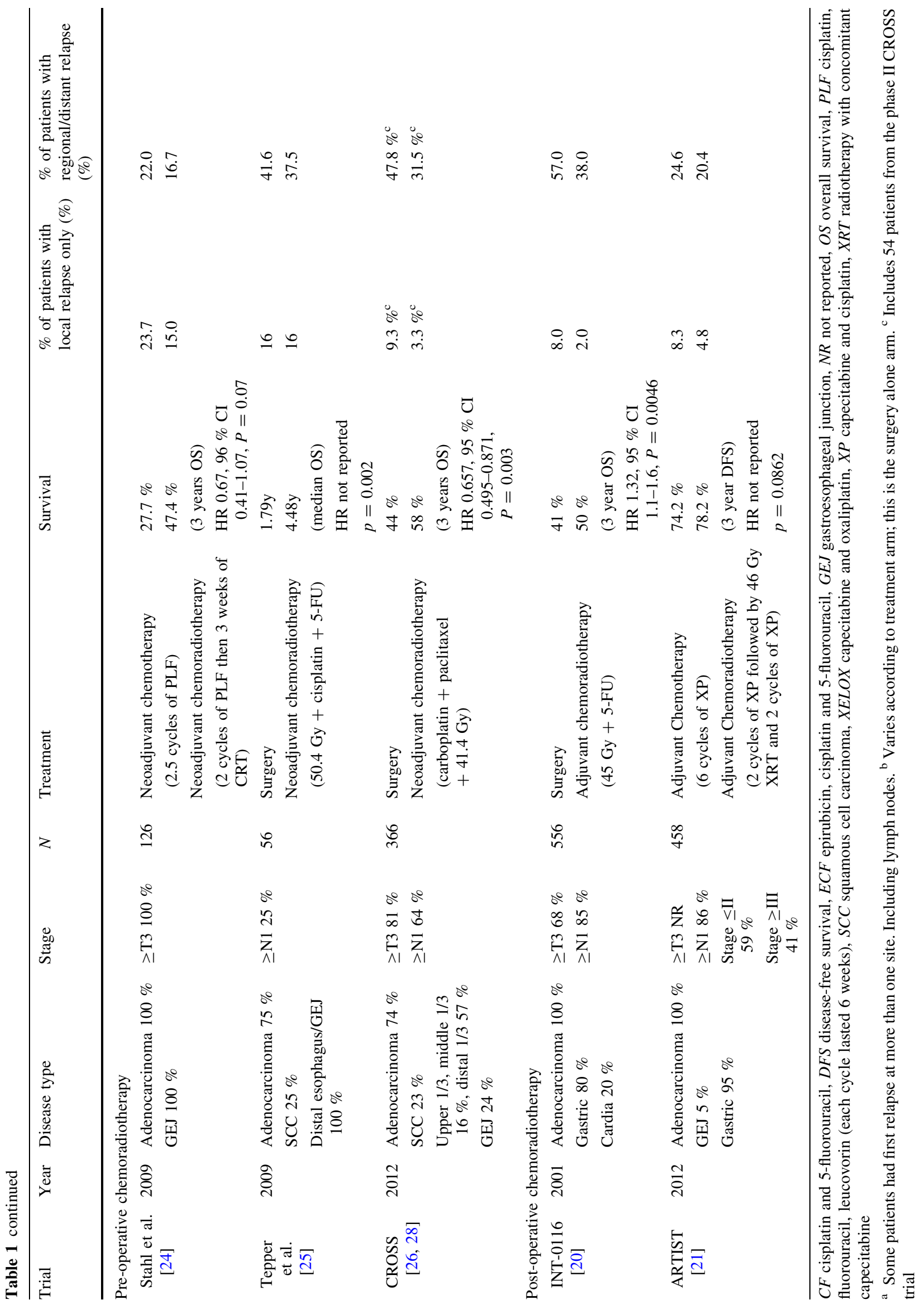


This review will focus on the treatment of operable esophagogastric adenocarcinoma, touching only briefly on SCC of the esophagus as this is considered sufficiently distinct from adenocarcinoma to merit a unique review. We will include a short discussion of the surgical treatment in addition to an overview of the evidence for adjuvant, perioperative and neoadjuvant treatment approaches (see Table 1) and conclude with an outline of the role of targeted therapies and ongoing trials.

\section{How geography and surgery impact on treatment choice}

Regardless of the choice of adjuvant or neoadjuvant therapy, surgery is a critical component of patients' treatment; radical surgery is indicated for stage IB-III (T1-3, N0-2, M0) gastric cancer and for localized or locally advanced esophageal cancer (T1-4, N0-1, M0) [4, 5]. Operative technique varies according to tumor site and extent; tumors extending into the distal esophagus frequently require a two-field transthoracic and transabdominal approach, which is associated with increased morbidity. Compared to esophageal cancer, gastric tumors are subject to a more extended lymph node dissection but the optimal extent of lymph node dissection for patients with gastric cancer has previously been the subject of some debate. Although D2 resection is now the recommended global standard, this may not performed routinely in Europe and the US outside of largevolume surgical centers and was certainly not standard practice when many trials used to define treatment paradigms were performed. Thus, whether a D2 resection has been or will be performed is very relevant to the choice of treatment for individual patients.

Distal gastric cancers have declined in incidence in Western countries; however, this remains the most prevalent anatomic subsite in Asian patients [6]. In contrast, lower esophageal cancers and those involving the GEJ and proximal stomach have increased rapidly in incidence in Western countries [7]. As tumor site influences the operative approach required, this results in Western patients frequently requiring more invasive surgery. This in turn impacts on postoperative morbidity and tolerance of adjuvant chemotherapy. Western patients also characteristically present with more locally advanced, bulky tumors, and accordingly a second benefit of a neoadjuvant approach is that preoperative tumor downstaging may reduce the risk of incomplete resections due to involved margins with proximal tumors. In contrast, margins are less often at risk in tumors of the body or antrum of the stomach, and distal subtotal gastrectomy for these tumors (even with D2 lymph node dissection) is associated with a less prolonged convalescent period rendering these patients more suitable for adjuvant therapy.

Finally, outcomes for Asian patients are usually significantly better than those seen in Western populations. For example, in the surgery-alone arms, the 3-year overall survival (OS) of patients in the Asian CLASSIC trial was $78 \%$, compared to $41 \%$ in the US INT-0116 trial, and 5-year OS in the ACTS-GC trial was $61 \%$ compared to $23 \%$ in the UK MAGIC trial [8-11]. Outcomes from trials conducted in Asian populations may therefore not be generalizable to those performed in Western populations and vice versa.

\section{Perioperative chemotherapy: a rational choice for gastric, esophageal and junctional tumors}

The UK MAGIC trial randomized 503 patients with adenocarcinoma of the stomach, GEJ or lower esophagus to either surgery alone or to surgery plus perioperative chemotherapy with epirubicin, cisplatin and 5-fluorouracil [10]. Three quarters of the patients in this study had gastric cancer; the remainder were distal esophageal (14\%) and junctional $(11 \%)$. A significant survival benefit was seen with perioperative chemotherapy compared to surgery alone (5-year survival rates of 36 versus $23 \%$ ), and this regimen became a standard of care, particularly in Europe [10]. Subgroup analyses demonstrate the benefit of perioperative chemotherapy to be present for all anatomic subsites but most pronounced for tumors of the GEJ. A major limitation of MAGIC is that only $38 \%$ of patients had a D2 resection, and so the benefits of perioperative chemotherapy in patients undergoing a D2 resection have not been confirmed.

The benefits of a perioperative approach are also demonstrated in the French FNCLCC/FFCD phase III trial, which randomized 224 patients to either perioperative chemotherapy with cisplatin and fluorouracil or to surgery alone [12]. This trial showed improved 5-year OS (38 versus $24 \%$, HR $0.69, P=0.02$ ) and disease-free survival (5 years rate 34 versus $19 \%$, HR $0.65, P=0.003$ ) in patients who received perioperative chemotherapy [12]. In contrast to MAGIC, this study contained a majority (75\%) of junctional and lower esophageal tumors. Therefore, perioperative chemotherapy has been shown to improve outcomes compared to surgery alone for both stomach and esophageal cancers.

Benefits of a perioperative approach include both tumor downstaging and superior preoperative tolerability of chemotherapy. Perioperative chemotherapy may lead to tumor shrinkage and thereby improve R0 resection rates. For example, preoperative chemotherapy in the MAGIC study improved rates of curative resection from 70.3 to $79.3 \%$ 
$(P=0.03)[10]$, and similar results were also seen in the French study (73 versus $84 \%, P=0.04$ ) [12]. In addition, although preoperative chemotherapy is well tolerated, a significant proportion of patients are not fit enough after surgery for adjuvant treatment. For example, in the FFCD and MAGIC trials, 87-91\% of patients completed preoperative treatment, but only $50-65.6 \%$ of patients started postoperative treatment and only $42-50 \%$ of patients completed postoperative treatment $[10,12]$.

Neoadjuvant chemotherapy alone, although an important component of the perioperative approach, has been less successful in improving survival for patients with resectable esophagagogastric cancer. The small $(N=144)$ EORTC 40954 trial randomized patients with gastric cancer and junctional cancers (in roughly equal proportions) to surgery alone or to preoperative chemotherapy with cisplatin and 5-fluorouracil followed by surgery. This trial showed a significantly increased rate of $\mathrm{R} 0$ resections with chemotherapy, but this did not translate into a survival benefit [13]. This trial had limited statistical power as there were a low number of events in both arms because of the better than expected surgical outcomes and early trial closure due to poor accrual. In addition, a high proportion of patients underwent a D2 resection (94\%), and this may have contributed to the improvement in survival and lack of benefit from chemotherapy in comparison with the MAGIC and FFCD trials.

The results of trials of neoadjuvant chemotherapy in esophageal cancer have also shown conflicting results. The largest trial in this setting is the MRC OE02 trial, which randomized 802 patients with esophageal cancer $(66 \%$ adenocarcinoma) to either surgery alone or two cycles of preoperative chemotherapy with cisplatin and 5-fluorouracil followed by surgery [14]. Even though patients only received two cycles of chemotherapy, this trial showed an improvement in 5-year survival with chemotherapy (23\% versus $17 \%, P=0.03$ ) [14]. This survival benefit was equivalent regardless of tumor histology [14]. However, these results are not consistent with those seen in the RTOG trial 8911, which was approximately equally split between squamous and adenocarcinoma. This large $(N=467)$ trial showed no difference in overall survival in patients with esophageal cancer who received neoadjuvant chemotherapy compared with patients who proceeded straight to surgery [15]. However, a meta-analysis reviewing eight trials of neoadjuvant chemotherapy for resectable esophagogastric cancer demonstrated a survival benefit for chemotherapy [HR $0.90(0.81-1.00) ; P=0.05]$. Interestingly, however, this benefit appears to be driven by the benefit derived by adenocarcinoma patients [HR 0.78 (0.64-0.95); $P=0.014]$, as the difference was non-significant for SCC [HR $0.88(0.75-1.03) ; P=0.12$ ] [16]. The results of the large OE05 trial (ClinicalTrials.gov identifier: NCT00041262), which randomized patients with adenocarcinoma of the esophagus/GEJ to either two cycles of neoadjuvant chemotherapy with cisplatin and fluorouracil or four cycles of ECX (epirubicin, cisplatin and capecitabine), are anticipated to be published soon and should provide further information on the optimal neoadjuvant chemotherapy approach.

\section{Adjuvant chemotherapy improves survival for adequately resected Asian gastric cancer patients}

Initial studies evaluating the use of adjuvant chemotherapy in gastric cancer compared to surgery alone did not demonstrate compelling results. However, a meta-analysis of 17 trials that closed to recruitment prior to 2004 demonstrated that adjuvant chemotherapy was associated with a statistically significant improvement in OS (HR 0.82; $95 \%$ CI $0.76-0.90 ; P \leq 0.001$ ) and disease-free survival (DFS) (HR $0.82 ; 95 \%$ CI $0.75-0.9 ; P \leq 0.001$ ), resulting in an improvement of 5-year OS from 49.6 to $55.3 \%$ [17]. The first convincing evidence from a randomized clinical trial for the benefit of adjuvant chemotherapy was first seen in the Asian Phase III Adjuvant Chemotherapy Trial of S-1 for Gastric Cancer (ACTS-GC) study [18], which led to adjuvant chemotherapy becoming the standard of care for resected gastric cancer patients in Asia. This study randomized 1,059 patients with D2 resected gastric cancer to either surgery alone or 1 year of adjuvant chemotherapy with the oral fluoropyrimidine S-1 [18]. Adjuvant chemotherapy was associated with an improvement in 5-year relapse-free (65.4 versus $53.1 \%$ ) and overall survival (71.7 versus $61.1 \%$ ) [11]. The gains in overall survival seen with these results are comparable to those seen using the perioperative chemotherapy or neoadjuvant chemoradiotherapy approaches used in Western populations. The use of S-1 is now a standard treatment in Asian patients; however, this drug is poorly tolerated in Western populations because of CYP2A6 polymorphisms and is therefore not widely used outside of Asia [11].

The subsequent phase III CLASSIC study randomized 1,035 Asian patients with gastric cancer to surgery alone (D2 resection) or to surgery plus eight cycles of adjuvant chemotherapy with capecitabine and oxaliplatin (XELOX) [8]. This study also showed a significant improvement in 3 -year DFS with chemotherapy (74 versus $59 \%$, HR 0.56 , $95 \%$ CI $0.44-0.72 ; P \leq 0.0001)$, with particular benefit seen in node-positive patients and an improvement in 5-year overall survival (78 versus $69 \%, P=0.0029$ ) [19]. Both ACTS-GC and CLASSIC almost exclusively contain patients with gastric cancers (100 and $98 \%$ respectively), and it is therefore difficult to extrapolate this treatment approach to patients with proximal tumors. In CLASSIC, 
XELOX was associated with greater grade 3 and 4 toxicities than S-1 in ACTS-GC (rates of grade 3/4 neutropenia/ leukopenia, vomiting and fatigue being 22 versus $1.2 \%$; 7 versus $1 \%$ and 5 versus $0.6 \%$, respectively), whereas 3-year DFS is almost identical between the studies (74\% in CLASSIC versus $72 \%$ in ACTS-GC) $[8,18]$. Only $67 \%$ of patients in CLASSIC completed 6 months of chemotherapy compared to $78 \%$ in ACTS-GC. Although the use of capecitabine and oxaliplatin in CLASSIC appears to sidestep the issue of geographic pharmacogenomic variability that $\mathrm{S} 1$ poses neatly, the use of adjuvant chemotherapy for resected gastric cancer has not been widely adopted outside of Asia because of a preference for preoperative tumor downstaging of more bulky Western tumors, regional variation in the extent of lymphadendectomy and the lack of evidence for this approach in proximal tumors.

\section{Adjuvant chemoradiotherapy: optimal if high risk of residual disease?}

Adjuvant chemoradiotherapy is a standard treatment option in the USA as a result of INT-0116, a large phase III trial comparing adjuvant chemoradiotherapy (45 Gy combined with 5-FU and leucovorin) with observation for adenocarcinoma of the stomach or GEJ [9]. This trial showed an improvement in survival in patients who received adjuvant chemoradiotherapy, with 3-year OS rates of $50 \%$ in the chemoradiotherapy group compared to $41 \%$ in patients treated with surgery alone [9]. An update demonstrated that the benefit from chemoradiotherapy has persisted after more than 10 years of follow-up, with hazard ratios for overall survival of 1.32 (95\% CI, 1.10-1.6; $P=0.0046$ ) and for relapse-free survival of 1.51 (95\% CI, 1.25-1.83; $P=0.001$ ) [20]. Approximately $80 \%$ of patients in INT0116 had gastric cancer; the remaining $20 \%$ were junctional tumors. Therefore, this approach may be reasonable for both distal and proximal esophagogastric cancers. However, a major concern regarding this trial is that $90 \%$ of patients had a limited lymph node dissection (D0 or D1), and therefore chemoradiotherapy may have merely compensated for insufficient resection [9]. This theory is supported by the results of the ARTIST trial [21].

The Asian ARTIST trial randomized 458 patients with curatively resected gastric cancer to either six cycles of postoperative XP (cisplatin and capecitabine) or two cycles of XP followed by chemoradiotherapy (45 Gy + capecitabine $1,650 \mathrm{mg} / \mathrm{m}^{2}$ per day for 5 weeks) and a further two cycles of XP [21]. All patients in this trial had a $\mathrm{D} 2$ resection, and therefore this study aimed to establish whether the benefits of chemoradiotherapy were also seen in patients who had an optimal resection.
Overall, this study did not show a significant improvement in DFS with the addition of chemoradiotherapy $(P=0.0862)$ [21]. One reason for this may be that approximately $60 \%$ of patients were stage IB-II in whom the relative benefit of any adjuvant approach is likely to be small [21]. However, patients with pathologic lymph node metastases at the time of surgery had improved DFS $(P=0.0365)$ with chemoradiotherapy, which suggests that these patients may represent a subgroup who derive benefit from chemoradiotherapy. As patients with more advanced nodal disease are more likely to have residual tumor even following D2 resection, this is congruent with the argument that adjuvant chemoradiotherapy is most effective in the setting of residual local disease (i.e., INT0116). Notably, there was a trend toward a lower local recurrence rate in the chemoradiotherapy arm of ARTIST (4.8 versus $8.3 \%$ for CRT versus chemotherapy, respectively), although this did not reach statistical significance. In conclusion, although there is evidence supporting the use of chemoradiotherapy over surgery alone for both gastric and a smaller numbers of junctional tumors, this benefit has not yet been confirmed in patients following D2 resection. This is being evaluated further in the ARTIST-II trial (ClinicalTrials.gov Identifier: NCT01761461).

\section{Neoadjuvant chemoradiotherapy comes in from the cold}

Neoadjuvant chemoradiotherapy for junctional tumors has recently undergone a renaissance. However, it is important to view recent trial results in the historical and histological context. Initial trials of neoadjuvant chemoradiotherapy showed inconsistent results; Walsh et al. randomized 113 patients with esophagogastric adenocarcinoma (65.5\% were middle/lower esophageal tumors and $34.5 \%$ tumors of the cardia) to surgery alone or to neoadjuvant chemoradiotherapy followed by surgery [22]. This study showed an improvement in median survival from 11 to 16 months with chemoradiotherapy [22]. However, these results are limited by the small number of patients, the extremely poor survival seen in the surgery alone arm (median survival of 11 months) and inadequate tumor staging by today's standards (CT, PET, EUS and laparoscopy were not mandated). A subsequent Australasian trial (62\% adenocarcinoma, $38 \%$ SCC) did not show any improvement in progression-free or overall survival with neoadjuvant chemoradiotherapy compared to surgery [23]. However, subgroup analysis suggested that patients with squamous tumors had better progression-free survival with chemoradiotherapy than non-squamous tumors (HR 0.47 versus 1.02) [23]. 
Two small studies that are used to support the role for chemoradiotherapy are those reported by Stahl et al. and CALGB 9781 [24, 25]. Stahl et al. [24] randomized 126 patients with adenocarcinoma of the lower esophagus or gastric cardia to either neoadjuvant chemotherapy followed by surgery or chemotherapy followed by chemoradiotherapy and then surgery. Although this study was underpowered because it closed prematurely because of low accrual, it did demonstrate an improvement in 3-year survival rates with chemotherapy plus chemoradiotherapy (47.4\%) compared to chemotherapy alone $(27.7 \%$ ) (log rank $P=0.07$ ) [24]. These results are in keeping with the small study $(N=56)$ by Tepper et al. [25], which also randomized a small number of patients $(75 \%$ adenocarcinoma) to triple modality therapy (chemoradiotherapy and surgery) or surgery alone. A significant benefit was seen for chemoradiotherapy patients (median OS 4.48 versus 1.79 years, $P=0.002$ ); however, no breakdown according to tumor histology was provided for these results.

Although the two aforementioned studies were small and underpowered, the phase III CROSS trial was not. This study randomized 366 patients with adenocarcinoma $(75 \%)$, SCC $(23 \%)$ or large-cell undifferentiated carcinoma $(2 \%)$ of the esophagus/GEJ to neoadjuvant treatment with weekly carboplatin and paclitaxel for 5 weeks with concurrent radiotherapy (41.4 Gy in 23 fractions, 5 days per week) or to surgery alone [26]. Most patients had tumors of the distal esophagus $(58 \%)$ or the GEJ (24\%). Tumors exceeding $8 \mathrm{~cm}$ in length and $5 \mathrm{~cm}$ in diameter were excluded from the study, as were tumors of the cardia with minimal invasion of the esophagus. The overall results of this study demonstrate a very significant improvement in OS with chemoradiotherapy (49.4 versus 24 months, $P=0.003$ ); however, this was strongly driven by the benefit seen in patients with SCC, and the adjusted hazard ratios were not significant for adenocarcinoma $(P=0.07)$ [26]. It is also notable that the control arm in the CROSS study was surgery alone. It is unclear whether the same benefits would be seen with a more contemporary control arm such as perioperative chemotherapy. This question is currently being assessed in an ongoing randomized clinical trial (ClinicalTrials.gov identifier: NCT01726452).

Pathological complete response (pCR) rates are significantly increased when radiation is used in conjunction with chemotherapy, and pCR rates are associated with improved survival in esophageal cancer [27]. However, pCR is much more common in radiosensitive SCC than adenocarcinoma. In CROSS, $49 \%$ of patients with SCC treated with chemoradiotherapy achieved a pCR compared to $23 \%$ of patients with adenocarcinoma $(P=0.008)$ [26]. Importantly, SCC patients achieving a sustained pCR may not need to undergo surgery. This is less often the case for patients with adenocarcinoma. Furthermore, although pCR is associated with an increased chance of an $\mathrm{R} 0$ resection, complete response is not mandatory to achieve a negative margin, and tumor downstaging alone may be sufficient. This is particularly true for adenocarcinoma, where perioperative chemotherapy is associated with relatively few complete tumor regressions, but still results in significant downstaging and increased $\mathrm{R} 0$ resections.

Chemoradiotherapy may also significantly reduce the risk of local recurrence; however, again this is more relevant for patients with SCC. For most patients, locally advanced esophagogastric adenocarcinoma is a systemic disease: following treatment the majority of patients who develop recurrent disease have distant metastases that chemoradiotherapy will not prevent (see Table 1). In contrast, chemotherapy appears to reduce the number of patients developing relapse at distant sites compared to surgery alone $[8,10,12]$. A combined analysis of the CROSS phase III trial and the preceding CROSS phase II trial (54 patients treated with chemoradiotherapy and surgery) showed that chemoradiotherapy reduced the risk of isolated local recurrence ( 3.3 versus $9.3 \%$ ) and peritoneal carcinomatosis $(4.2$ versus $13.7 \%, P<0.001)$ [28]. However, although chemoradiotherapy reduced the risk of hematogenous metastases (28.6 versus $35.4 \%, P=0.03$ ), distant failure remained the most common form of disease recurrence [28]. In addition, it is notable that adjusted hazard ratios demonstrate no improvement in survival for patients with node-positive disease (those at increased risk of systemic metastases) [26]. That improving local control may not translate into a survival advantage for patients with adenocarcinoma is also supported by the results from the MUNICON II trial, in which patients with gastroesophageal adenocarcinoma who did not respond on PET to initial chemotherapy were treated with salvage radiotherapy. Although R0 resection rates were improved by the addition of radiotherapy, survival was not improved because of the high rate of occurrence of systemic metastatic disease $[29,30]$.

\section{FDG-PET directed therapy}

Many esophagogastric adenocarcinomas exhibit significant levels of FDG-uptake on PET-CT; however, this is less common in tumors of Lauren's diffuse subtype [31]. Patients who do not demonstrate a reduction in SUV on FDG-PET following one cycle of chemotherapy have inferior outcomes than those demonstrating a metabolic response, regardless of whether the patient continues with neoadjuvant chemotherapy or proceeds directly to surgery $[32,33]$. As noted above, for metabolically nonresponding patients, salvage chemoradiotherapy may 
Table 2 Suggested approach to the treatment of operable esophagogastric cancer

\begin{tabular}{|c|c|c|}
\hline Tumor type & Suggested treatment & Rationale \\
\hline \multicolumn{3}{|c|}{ Lower esophageal and junctional tumors } \\
\hline \multirow[t]{2}{*}{$\mathrm{SCC}$} & \multirow[t]{2}{*}{ Chemoradiotherapy } & $\begin{array}{l}58 \% \text { reduction in risk of } \\
\text { death with CRT in } \\
\text { CROSS for SCC (HR } \\
0.422, P=0.007)\end{array}$ \\
\hline & & $\begin{array}{l}\text { No significant reduction } \\
\text { in risk of death in AC } \\
\text { in CROSS (HR 0.741, } \\
P=0.07 \text { ) }\end{array}$ \\
\hline \multirow[t]{6}{*}{ Adenocarcinoma } & \multirow[t]{4}{*}{$\begin{array}{l}\text { Perioperative } \\
\text { chemotherapy }\end{array}$} & $\begin{array}{l}\text { Significant downsizing } \\
\text { with perioperative } \\
\text { chemotherapy } \\
\text { increased R0 resection } \\
\text { rate }\end{array}$ \\
\hline & & $\begin{array}{l}\text { Concurrent treatment of } \\
\text { micrometastatic } \\
\text { disease }\end{array}$ \\
\hline & & $\begin{array}{l}\text { Chemotherapy better } \\
\text { tolerated in } \\
\text { neoadjuvant setting for } \\
\text { patients undergoing } \\
\text { transthoracic } \\
\text { esophagogastrectomy } \\
\text { or proximal } \\
\text { gastrectomy }\end{array}$ \\
\hline & & $\begin{array}{l}26-31 \% \text { statistically } \\
\text { significant reduction in } \\
\text { risk of death with } \\
\text { MAGIC/FFCD } \\
\text { regimens }\end{array}$ \\
\hline & \multirow[t]{2}{*}{ Chemoradiotherapy } & $\begin{array}{l}\text { May reduce the risk of } \\
\text { local recurrence }\end{array}$ \\
\hline & & $\begin{array}{l}\text { Can result in significant } \\
\text { downsizing, some } \\
\text { pathological complete } \\
\text { responses and increase } \\
\text { R0 resection rates, } \\
\text { although this may not } \\
\text { translate to an } \\
\text { improvement in } \\
\text { survival due to the high } \\
\text { rate of systemic } \\
\text { metastatic disease }\end{array}$ \\
\hline \multicolumn{3}{|c|}{ Distal gastric adenocarcinoma } \\
\hline \multirow[t]{2}{*}{ Western patients } & \multirow[t]{2}{*}{$\begin{array}{l}\text { Perioperative } \\
\text { chemotherapy }\end{array}$} & $\begin{array}{l}\text { Significant downsizing } \\
\text { of tumor and improved } \\
\text { resection rate leading } \\
\text { to improvements in OS } \\
\text { as above }\end{array}$ \\
\hline & & $\begin{array}{l}\text { No evidence for adjuvant } \\
\text { chemotherapy } \\
\text { approach in Western } \\
\text { patients }\end{array}$ \\
\hline
\end{tabular}

improve local control and improve resection rates, but does not impact on overall survival because of high rates of systemic metastatic disease [30]. Although PET-
Table 2 continued

\begin{tabular}{|c|c|c|}
\hline Tumor type & Suggested treatment & Rationale \\
\hline \multirow{6}{*}{$\begin{array}{c}\text { East Asian } \\
\text { patients }\end{array}$} & \multirow{4}{*}{$\begin{array}{l}\text { Postoperative } \\
\text { chemoradiotherapy }\end{array}$} & Limited indications: \\
\hline & & $\begin{array}{l}\text { Patient under-staged } \\
\text { prior to surgical } \\
\text { resection }\end{array}$ \\
\hline & & $\begin{array}{l}\text { No neoadjuvant } \\
\text { chemotherapy received }\end{array}$ \\
\hline & & $\begin{array}{l}\text { Local control at risk } \\
\text { (R1 resection, }<\mathrm{D} 2 \\
\text { resection) }\end{array}$ \\
\hline & \multirow[t]{2}{*}{$\begin{array}{l}\text { Postoperative } \\
\text { adjuvant } \\
\text { chemotherapy }\end{array}$} & $\begin{array}{l}\text { Good evidence for } \\
\text { improvements in OS in } \\
\text { optimally resected } \\
\text { Asian patients }\end{array}$ \\
\hline & & $\begin{array}{l}\text { XELOX may be superior } \\
\text { in a subset of } \mathrm{N} 2 \\
\text { disease (HR } 0.45 \text { vs. } \\
0.779 \text { ) but not N0/1 } \\
\text { disease (HR } 0.9 \text { versus } \\
0.317 \text { for N0 disease } \\
\text { and } 0.62 \text { vs. } 0.606 \text { for } \\
\text { N1 disease) }\end{array}$ \\
\hline
\end{tabular}

$\overline{A C \text { adenocarcinoma, } C R T \text { chemoradiotherapy, } H R \text { hazard ratio, } O S}$ overall survival, $S C C$ squamous cell carcinoma, $X E L O X$ capecitabine and oxaliplatin

directed therapy appears an attractive choice for selecting chemosensitive patients and avoiding the toxicity of futile therapy in metabolic non-responders, these studies are limited by the small numbers of patients enrolled and are thus underpowered to be used for evidence-based therapy selection.

\section{Future directions}

Based on the evidence above, there are significant unanswered questions regarding the management of patients with operable OG cancer. There are a number of currently recruiting studies that may help to clarify the optimal treatment strategy for these patients. For example, the phase III Chemoradiotherapy after Induction Chemotherapy of Cancer in the Stomach (CRITICS) trial (ClinicalTrials.gov Identifier: NCT00407186) is currently investigating preoperative chemotherapy with epirubicin, cisplatin and capecitabine (ECX) followed by either surgery and a further three cycles of ECX or surgery and chemoradiotherapy (45 Gy, cisplatin and capecitabine). This study will therefore hopefully answer the question as to whether postoperative chemoradiotherapy will improve the current European standard of perioperative chemotherapy. Further information on the chemotherapy versus chemoradiotherapy debate may also be provided by the 
MAGIC versus CROSS trial (ClinicalTrials.gov identifier: NCT01726452).

\section{Moving forward: integration of targeted therapy into the perioperative setting}

There has been increasing recognition of the potential benefit of therapies targeting molecular characteristics such as HER2, MET and FGFR in the advanced disease setting. HER2 is currently the most well-established target following the results of the ToGA trial, which showed that the addition of trastuzumab to chemotherapy improved overall survival in patients with advanced gastric/GEJ cancer [34]. The use of trastuzumab has not yet however been established in the neoadjuvant or adjuvant setting, and this is currently being evaluated in various clinical trials. For example, the TOXAG study (ClinicalTrials.gov identifier: NCT01748773) aims to assess the safety and tolerability of adding trastuzumab to adjuvant chemoradiotherapy with oxaliplatin and capecitabine in patients with HER2 positive gastric/GEJ cancers, and an American study (Clinical trials.gov identifier: NCT01196390) is investigating the addition of trastuzumab to neoadjuvant chemoradiotherapy with paclitaxel and carboplatin. Other anti-HER2 therapies are also being investigated, e.g., a sub-study of the ST03 trial (ClinicalTrials.gov identifier: NCT00450203) is investigating the feasibility of lapatinib (an anti-HER2/ EGFR tyrosine kinase inhibitor) in combination with perioperative chemotherapy. It is hoped that the addition of appropriately targeted anti-HER 2 directed therapy into the perioperative setting will yield survival benefits comparable to those seen in other HER2 amplified malignancies.

\section{Can there be consensus?}

Given the heterogeneity of patient characteristics and treatment paradigms that define the perioperative approach to resectable esophagogastric cancer, it is difficult to reach a consensus opinion on this issue, but a suggested approach is shown in Table 2. For squamous cell carcinoma of the esophagus, the answer is clear; combination chemoradiotherapy plus or minus surgical resection results in marked gains in overall survival. For patients with resectable true gastric cancers, there is ample choice in perioperative treatment. While optimally resected Asian patients clearly benefit from adjuvant chemotherapy with $\mathrm{S} 1$ or XELOX, this approach has not been proven in patients undergoing D1 resections, Western populations or junctional tumors. Gastric cancer patients with more bulky tumors, in common with patients with distal esophageal or junctional tumors, benefit from downstaging delivered by a perioperative treatment approach. Postoperatively, and in particular in the setting of suboptimal resection, adjuvant chemoradiation may improve survival. Neoadjuvant chemoradiotherapy may provide significant downstaging for more GEJ tumors, and this may be important for patients with threatened margins. However, as most adenocarcinomas recur systemically, adequate chemotherapy dose intensity is also essential for control of systemic disease.

Acknowledgments We acknowledge support from the NIHR RM/ ICR Biomedical Research Centre.

Conflict of interest DC has received research funding from Amgen, Roche, Sanofi-Aventis, Merck-Serono, Novartis and Celgene, and has had advisory roles (uncompensated) with Roche and Amgen. SYM and ES have declared no conflicts of interest.

\section{References}

1. Lauren $P$. The two main histological types of gastic carcinoma. Acta Pathol Microbiol Scand. 1965;64:31-49 Epub 1965/01/01.

2. Marrelli D, Roviello F, de Manzoni G, Morgagni P, Di Leo A, Saragoni L, et al. Different patterns of recurrence in gastric cancer depending on Lauren's histological type: longitudinal study. World J Surg. 2002;26(9):1160-5.

3. Tan IB, Ivanova T, Lim KH, Ong CW, Deng N, Lee J, et al. Intrinsic subtypes of gastric cancer, based on gene expression pattern, predict survival and respond differently to chemotherapy. Gastroenterology. 2011;141(2):476-85. doi:10.1053/j.gastro. 2011.04.042.

4. Okines A, Verheij M, Allum W, Cunningham D, Cervantes A. Gastric cancer: ESMO Clinical Practice Guidelines for diagnosis, treatment and follow-up. Ann Oncol: Off J Eur Soc Med Oncol/ ESMO. 2010;21(Suppl 5):v50-4. doi:10.1093/annonc/mdq164 (PubMed PMID: 20555102).

5. Stahl M, Budach W, Meyer HJ, Cervantes A. Esophageal cancer: clinical practice guidelines for diagnosis, treatment and followup. Ann Oncol: Off J Eur Soc Med Oncol/ESMO. 2010;21(Suppl 5):v46-9. doi:10.1093/annonc/mdq163.

6. Bertuccio P, Chatenoud L, Levi F, Praud D, Ferlay J, Negri E, et al. Recent patterns in gastric cancer: a global overview. Int J Cancer. 2009;125(3):666-73.

7. Wu H, Rusiecki JA, Zhu K, Potter J, Devesa SS. Stomach carcinoma incidence patterns in the united states by histologic type and anatomic site. Cancer Epidemiol Biomark Prev. 2009;18(7):1945-52. doi:10.1158/1055-9965.epi-09-0250.

8. Bang YJ, Kim YW, Yang HK, Chung HC, Park YK, Lee KH, et al. Adjuvant capecitabine and oxaliplatin for gastric cancer after D2 gastrectomy (CLASSIC): a phase 3 open-label, randomised controlled trial. Lancet. 2012;379(9813):315-21. doi:10. 1016/s0140-6736(11)61873-4 (Epub 2012/01/10 PubMed PMID: 22226517)

9. Macdonald JS, Smalley SR, Benedetti J, Hundahl SA, Estes NC, Stemmermann GN, et al. Chemoradiotherapy after surgery compared with surgery alone for adenocarcinoma of the stomach or gastroesophageal junction. New Engl J Med. 2001; 345(10):725-30. doi:10.1056/NEJMoa010187 (Epub 2001/09/08, PubMed PMID: 11547741).

10. Cunningham D, Allum WH, Stenning SP, Thompson JN, Van de Velde CJ, Nicolson M, et al. Perioperative chemotherapy versus 
surgery alone for resectable gastroesophageal cancer. New Engl J Med. 2006;355(1):11-20. doi:10.1056/NEJMoa055531.

11. Sasako M, Sakuramoto S, Katai H, Kinoshita T, Furukawa H, Yamaguchi T, et al. Five-year outcomes of a randomized phase III trial comparing adjuvant chemotherapy with S-1 versus surgery alone in stage II or III gastric cancer. J Clin Oncol: Off J Am Soc Clin Oncol. 2011;29(33):4387-93. doi:10.1200/jco.2011.36. 5908 (Epub 2011/10/20, PubMed PMID: 22010012).

12. Ychou M, Boige V, Pignon JP, Conroy T, Bouche O, Lebreton G, et al. Perioperative chemotherapy compared with surgery alone for resectable gastroesophageal adenocarcinoma: an FNCLCC and FFCD multicenter phase III trial. J Clin Oncol: Off J Am Soc Clin Oncol. 2011;29(13):1715-21. doi:10.1200/JCO.2010.33. 0597.

13. Schuhmacher C, Gretschel S, Lordick F, Reichardt P, Hohenberger W, Eisenberger $\mathrm{CF}$, et al. Neoadjuvant chemotherapy compared with surgery alone for locally advanced cancer of the stomach and cardia: European Organisation for Research and Treatment of Cancer randomized trial 40954. J Clin Oncol: Off J Am Soc Clin Oncol. 2010;28(35):5210-8. doi:10.1200/JCO. 2009.26.6114 (PubMed PMID: 21060024; PubMed Central PMCID: PMC3020693).

14. Allum WH, Stenning SP, Bancewicz J, Clark PI, Langley RE. Long-term results of a randomized trial of surgery with or without preoperative chemotherapy in esophageal cancer. J Clin Oncol. 2009;27(30):5062-7. doi:10.1200/jco.2009.22.2083.

15. Kelsen DP, Winter KA, Gunderson LL, Mortimer J, Estes NC, Haller DG, et al. Long-term results of RTOG trial 8911 (USA Intergroup 113): a random assignment trial comparison of chemotherapy followed by surgery compared with surgery alone for esophageal cancer. J Clin Oncol: Off J Am Soc Clin Oncol. 2007;25(24):3719-25. doi:10.1200/JCO.2006.10.4760.

16. Gebski V, Burmeister B, Smithers BM, Foo K, Zalcberg J, Simes J. Survival benefits from neoadjuvant chemoradiotherapy or chemotherapy in oesophageal carcinoma: a meta-analysis. Lancet Oncol. 2007;8(3):226-34. doi:10.1016/s1470-2045(07)70039-6.

17. Paoletti X, Oba K, Burzykowski T, Michiels S, Ohashi Y, Pignon JP, et al. Benefit of adjuvant chemotherapy for resectable gastric cancer: a meta-analysis. JAMA: J Am Med Assoc. 2010; 303(17):1729-37. doi:10.1001/jama.2010.534 (Epub 2010/05/06, PubMed PMID: 20442389).

18. Sakuramoto S, Sasako M, Yamaguchi T, Kinoshita T, Fujii M, Nashimoto A, et al. Adjuvant chemotherapy for gastric cancer with S-1, an oral fluoropyrimidine. New Engl J Med. 2007; 357(18):1810-20. doi:10.1056/NEJMoa072252 (Epub 2007/11/ 06, PubMed PMID: 17978289).

19. Noh SH, Park SR, Yang H-K, Chung HC, Chung I-J, Lee KH, et al. Adjuvant capecitabine and oxaliplatin (XELOX) for gastric cancer after D2 gastrectomy: final results from the CLASSIC trial. Ann Oncol. 2013;24(Suppl 4):iv14. doi:10.1093/annonc/ mdt201.7.

20. Smalley SR, Benedetti JK, Haller DG, Hundahl SA, Estes NC, Ajani JA, et al. Updated analysis of SWOG-directed intergroup study 0116: a phase III trial of adjuvant radiochemotherapy versus observation after curative gastric cancer resection. J Clin Oncol. 2012;. doi:10.1200/jco.2011.36.7136.

21. Lee J, Lim DH, Kim S, Park SH, Park JO, Park YS, et al. Phase III trial comparing capecitabine plus cisplatin versus capecitabine plus cisplatin with concurrent capecitabine radiotherapy in completely resected gastric cancer with $\mathrm{D} 2$ lymph node dissection: the ARTIST trial. J Clin Oncol. 2012;30(3):268-73. doi:10. 1200/jco.2011.39.1953.

22. Walsh TN, Noonan N, Hollywood D, Kelly A, Keeling N, Hennessy TP. A comparison of multimodal therapy and surgery for esophageal adenocarcinoma. New Engl J Med. 1996;335(7): 462-7. doi:10.1056/NEJM199608153350702.
23. Burmeister BH, Smithers BM, Gebski V, Fitzgerald L, Simes RJ, Devitt $\mathrm{P}$, et al. Surgery alone versus chemoradiotherapy followed by surgery for resectable cancer of the oesophagus: a randomised controlled phase III trial. Lancet Oncol. 2005;6(9):659-68. doi:10.1016/S1470-2045(05)70288-6.

24. Stahl M, Walz MK, Stuschke M, Lehmann N, Meyer H-J, RieraKnorrenschild J, et al. Phase III comparison of preoperative chemotherapy compared with chemoradiotherapy in patients with locally advanced adenocarcinoma of the esophagogastric junction. J Clin Oncol. 2009;27(6):851-6. doi:10.1200/jco.2008.17. 0506.

25. Tepper J, Krasna MJ, Niedzwiecki D, Hollis D, Reed CE, Goldberg R, et al. Phase III trial of trimodality therapy with cisplatin, Fluorouracil, radiotherapy, and surgery compared with surgery alone for esophageal cancer: CALGB 9781. J Clin Oncol. 2008;26(7):1086-92. doi:10.1200/jco.2007.12.9593.

26. van Hagen P, Hulshof MCCM, van Lanschot JJB, Steyerberg EW, Henegouwen MIVB, Wijnhoven BPL, et al. Preoperative chemoradiotherapy for esophageal or junctional cancer. New Engl J Med. 2012;366(22):2074-84.

27. van Hagen P, Wijnhoven BP, Nafteux P, Moons J, Haustermans $\mathrm{K}$, De Hertogh G, et al. Recurrence pattern in patients with a pathologically complete response after neoadjuvant chemoradiotherapy and surgery for oesophageal cancer. Br J Surg. 2013;100(2):267-73. doi:10.1002/bjs.8968.

28. Oppedijk V, van der Gaast A, van Lanschot JJ, van Hagen P, van Os R, van Rij CM, et al. Patterns of recurrence after surgery alone versus preoperative chemoradiotherapy and surgery in the CROSS trials. J Clin Oncol: Off J Am Soc Clin Oncol. 2014;. doi:10.1200/JCO.2013.51.2186.

29. zum Buschenfelde CM, Herrmann K, Schuster T, Geinitz H, Langer R, Becker K, et al. (18)F-FDG PET-guided salvage neoadjuvant radiochemotherapy of adenocarcinoma of the esophagogastric junction: the MUNICON II trial. J Nucl Med. 2011;52(8):1189-96. doi:10.2967/jnumed.110.085803 (Epub 2011/07/19, PubMed PMID: 21764790).

30. Krause BJ, Herrmann K, Wieder H, zum Buschenfelde CM. 18FFDG PET and 18F-FDG PET/CT for assessing response to therapy in esophageal cancer. J Nucl Med. 2009;50(Suppl 1):89-96. doi:10.2967/jnumed.108.057232.

31. Smyth E, Schoder H, Strong VE, Capanu M, Kelsen DP, Coit $\mathrm{DG}$, et al. A prospective evaluation of the utility of 2-deoxy-2[(18) F] fluoro-D-glucose positron emission tomography and computed tomography in staging locally advanced gastric cancer. Cancer. 2012;. doi:10.1002/cncr.27550 (Epub 2012/05/03, PubMed PMID: 22549558).

32. Ott K, Weber WA, Lordick F, Becker K, Busch R, Herrmann K, et al. Metabolic imaging predicts response, survival, and recurrence in adenocarcinomas of the esophagogastric junction. J Clin Oncol. 2006;24(29):4692-8. doi:10.1200/jco.2006.06.7801.

33. Lordick F, Ott K, Krause B-J, Weber WA, Becker K, Stein HJ, et al. PET to assess early metabolic response and to guide treatment of adenocarcinoma of the oesophagogastric junction: the MUNICON phase II trial. Lancet Oncol. 2007;8(9):797-805. doi:10.1016/S1470-2045(07)70244-9.

34. Bang Y-J, Van Cutsem E, Feyereislova A, Chung HC, Shen L, Sawaki A, et al. Trastuzumab in combination with chemotherapy versus chemotherapy alone for treatment of HER2-positive advanced gastric or gastro-oesophageal junction cancer (ToGA): a phase 3, open-label, randomised controlled trial. Lancet. 2010;376(9742):687-97. doi:10.1016/s0140-6736(10)61121-x.

35. Kelsen DP, Ginsberg R, Pajak TF, Sheahan DG, Gunderson L, Mortimer J, et al. Chemotherapy followed by surgery compared with surgery alone for localized esophageal cancer. New Engl J Med. 1998;339(27):1979-84. doi:10.1056/NEJM19981231 3392704. 